\title{
Nucleosynthesis of the Elements in Faint Supernovae and Hypernovae
}

\author{
Ken'ichi Nomoto ${ }^{1,2}$, Takashi Moriya ${ }^{2,1}$, and Nozomu Tominaga ${ }^{3,1}$ \\ ${ }^{1}$ Institute for the Physics and Mathematics of the Universe (IPMU), University of Tokyo \\ Kashiwanoha 5-1-5, Kashiwa, Chiba 277-8583, Japan \\ email: nomoto@astron.s.u-tokyo.ac.jp \\ ${ }^{2}$ Department of Astronomy, University of Tokyo, Bunkyo-ku, Tokyo 113-0033, Japan \\ ${ }^{3}$ Department of Physics, Konan University, Okamoto, Kobe 658-8501, Japan
}

\begin{abstract}
We review the properties of supernovae (SNe) as a function of the progenitor's mass $M$. (1) $M_{\text {up }}-10 M_{\odot}$ stars are super-AGB stars and resultant electron capture SNe may be Faint supernovae like Type IIn SN 2008S. (2) 10 - $12 M_{\odot}$ stars undergo Fe-core collapse to form neutron stars (NSs) and Faint supernovae. (3) $12 M_{\odot}-M_{\mathrm{BN}}$ stars undergo Fe-core collapse to form NSs and normal core-collapse supernovae. (4) $M_{\mathrm{BN}}-90 M_{\odot}$ stars undergo Fe-core collapse to form Black Holes. Resultant supernovae are bifurcate into Hypernovae and Faint supernovae. The observed properties of SN 2008ha can be explained with this type of Faint supernovae. (5) $90-140 M_{\odot}$ stars produce Luminous SNe, like SNe 2007bi and 2006gy. (6) $140-300 M_{\odot}$ stars become pair-instability supernovae which could be Luminous supernovae (SNe 2007bi and 2006gy). (7) Very massive stars with $M \gtrsim 300 M_{\odot}$ undergo core-collapse to form intermediate mass black holes. Some SNe could be more Luminous supernovae (like SN 2006gy).
\end{abstract}

Keywords. Galaxy: halo - gamma rays: bursts - nuclear reactions, nucleosynthesis, abundances — stars: abundances — stars: AGB — supernovae: general

\section{Faint Supernovae, Luminous Supernovae, and Hypernovae}

The final stages of massive star evolution, supernova properties, and their chemical yields depend on the progenitor's main-sequence masses $M$ (e.g., Arnett 1996, Smartt 2009). Here we call some specific supernovae ( $\mathrm{SNe}$ ) as follows. In terms of the kinetic explosion energy $E$, we use "Hypernovae" for such energetic SNe as $E_{51}=E / 10^{51} \mathrm{erg}$ $>$ 10. In terms of brightness, we use "Faint SNe (FSNe)" for low luminosity SNe, and "Luminous SNe (LSNe)" for SNe brighter than, say, -20 mag at maximum. The following mass ranges are set by various types of criteria, based on some combinations of observations and models. But the criteria and critical masses are not quite systematic yet, and should still be regarded as working hypothesis.

(1) $M_{\text {up }}-10 M_{\odot}$ stars: Faint supernovae (FSNe): These stars become electron capture SNe because their degenerate $\mathrm{O}+\mathrm{Ne}+\mathrm{Mg}$ cores collapse due to electron capture. $M_{\text {up }} \sim 9 \pm 0.5 M_{\odot}$ depending on the mass loss rate on the super-AGB phase thus on the metallicity (e.g., Pumo et al. 2009).

(2) 10 - $12 M_{\odot}$ stars: Faint SNe (FSNe): These stars undergo Fe-core collapse to form a neutron star (NS) after the phase of strong Neon shell-flashes (Nomoto \& Hashimoto 1988). Their Fe core is relatively small, and the resultant SNe tend to be faint (Smartt 2009).

(3) $12 M_{\odot}-M_{\mathrm{BN}}$ stars: Normal SNe: These stars undergo Fe-core collapse to form a NS, and produce significant amount of heavy elements from $\alpha$-elements and Fe-peak elements. The boundary mass between the NS and BH formation, $M_{\mathrm{BN}} \sim 25 M_{\odot}$, is only tentative. 
(4) $M_{\mathrm{BN}}$ - $90 M_{\odot}$ stars: Hypernovae and FSNe: These stars undergo Fe-core collapse to form a black hole (BH). SNe seem to be bifurcate into two branches, Hypernovae and Faint SNe. If the BH has little angular momentum, little mass ejection would take place and be observed as FSNe. On the other hand, a rotating BH could eject a matter in a form of jets to make a Hypernova. The latter explosions produce a large amount of heavy elements from $\alpha$-elements and Fe-peak elements. Nucleosynthesis in these jet-induced explosions is in good agreement with the abundance patterns observed in extremely metal-poor stars.

(5) 90 - $140 M_{\odot}$ stars: Luminous SNe (LSNe): These massive stars undergo nuclear instabilities and associated pulsations ( $\epsilon$-mechanism) at various nuclear burning stages depending on the mass loss and thus metallicity. Eventually, these stars undergo Fe-core collapse. Depending on the angular momentum, Hypernova-like energetic SNe could occur to produce large amount ${ }^{56} \mathrm{Ni}$. (Because of the large ejecta mass, the expansion velocities may not be high enough to form a broad line features.) Thanks to the large $E$ and ${ }^{56} \mathrm{Ni}$ mass, these SNe could be LSNe. The possible presence of circumstellar matter (CM) leads to energetic SN IIn. Pulsation could also cause luminous event.

(6) 140 - $300 M_{\odot}$ stars: LSNe: If these very massive stars (VMS) do not lose much mass, they become pair-instability supernovae (PISN). The star is completely disrupted without forming a $\mathrm{BH}$ and thus ejects a large amount of heavy elements, especially ${ }^{56} \mathrm{Ni}$. Radioactive decays could produce LSNe.

(7) Stars with $M \gtrsim 300 M_{\odot}$ : LSNe: These VMSs are too massive to be disrupted by PISN but undergo core collapse (CVMS), forming intermediate-mass black holes (IMBHs). Some mass ejection could be possible, associated with the possible jet-induced explosion, which becomes a very luminous SNe (LSNe).

In the following sections, we summarize the properties of the above supernovae in some detail. For their chemical yields, see Nomoto et al. (2009) for a recent review.

\section{8 - $10 M_{\odot}$ Super-AGB Progenitors and Faint Supernovae}

An $\mathrm{O}+\mathrm{Ne}+\mathrm{Mg}$ white dwarf is formed from $8 M_{\odot}-\mathrm{M}_{\text {up }}$ stars, where $\mathrm{M}_{\text {up }} \sim 9 \pm 0.5 M_{\odot}$ being smaller for smaller metallicity (Pumo et al. 2009).

For $\mathrm{M}_{\text {up }}-10 M_{\odot}$ stars, the core mass grows to $1.38 M_{\odot}$ and electron captures ${ }^{24} \mathrm{Mg}\left(\mathrm{e}^{-}, \nu\right){ }^{24} \mathrm{Na}\left(\mathrm{e}^{-}, \nu\right){ }^{20} \mathrm{Ne}$ and ${ }^{20} \mathrm{Ne}\left(\mathrm{e}^{-}, \nu\right){ }^{20} \mathrm{~F}\left(\mathrm{e}^{-}, \nu\right){ }^{20} \mathrm{O}$ induce collapse (Nomoto 1984).

The resultant explosion is induced by neutrino heating, and weak with the kinetic energy of as low as $E \sim 10^{50}$ erg (Kitaura et al. 2006). These stars produce little $\alpha$ elements and Fe-peak elements, but are important sources of $\mathrm{Zn}$ and light p-nuclei. These AGB supernovae may constitute an SN 2008S-like sub-class of Type IIn supernovae.

Nucleosynthesis in the supernova explosion of the $9 M_{\odot}$ star is as follows (Wanajo et al. 2009). The largest overproduction is shared by ${ }^{64} \mathrm{Zn},{ }^{70} \mathrm{Se}$, and ${ }^{78} \mathrm{Kr}$. The ${ }^{64} \mathrm{Zn}$ production provides an upper limit to the occurrence of exploding O-Ne-Mg cores at about $20 \%$ of all core-collapse supernovae. The ejecta mass of ${ }^{56} \mathrm{Ni}$ is $0.002-0.004 M_{\odot}$, much smaller than $\sim 0.1 M_{\odot}$ in more massive progenitors.

The expected small amount of ${ }^{56} \mathrm{Ni}$ as well as the low explosion energy of electron capture supernovae have been proposed as an explanation of the observed properties of Faint SNe of type IIn, such as SN 2008S and similar transients (Prieto et al. 2008; Thompson et al. 2009). The envelope of the AGB star is carbon-enhanced (Nomoto 1984). Then dust could easily be formed in mass loss. This may result in a deeply dust-enshrouded object such as the progenitor of SN 2008S (Prieto et al. 2008; Thompson et al. 2009). 

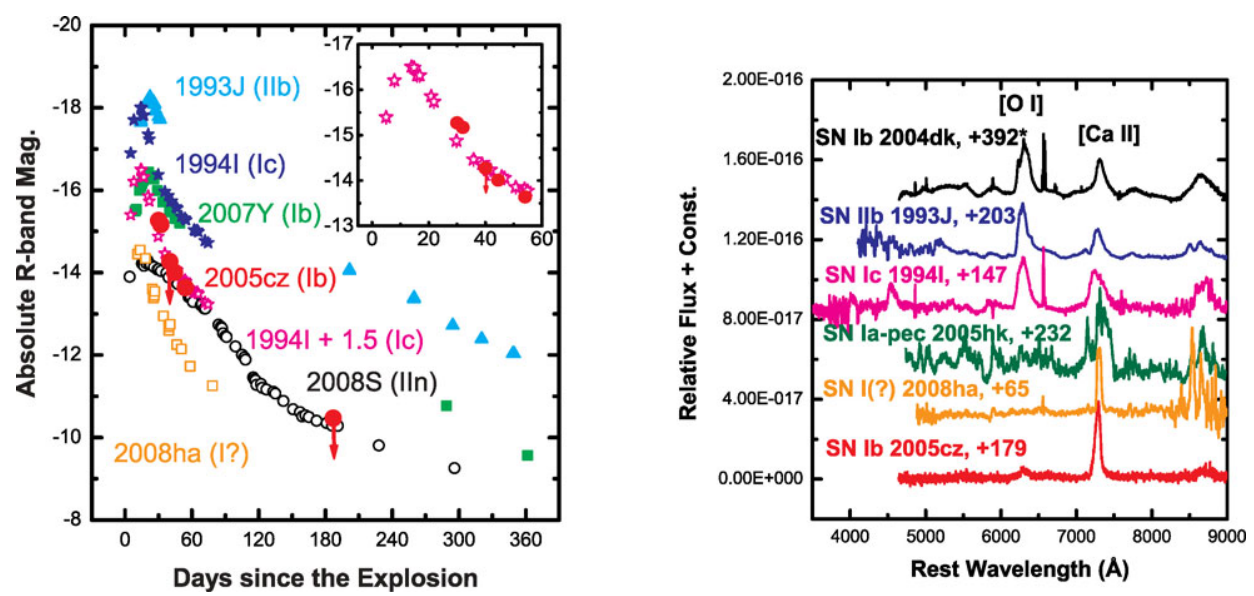

Figure 1. (Left): The absolute $R$-band light curve of faint supernovae: SN IIn 2008S (black open circles), SN Ib 2005cz (red circles), SN I 2008ha (orange open squares), and SN Ib 2007Y (green squares) as compared with those of SN IIb 1993J (cyan triangles) and SN Ic 1994I (blue stars). Also shown is the light curve of SN 1994I, but dimmed by 1.5 magnitudes (magenta open stars).

Figure 2. (Right): The late-time spectrum of SN Ib 2005cz $(t=+179$ days). Also shown are SN Ib 2004dk at $t \sim 390$ days, SN IIb 1993J at $t=+203$ days, SN Ic 1994I at $t=+147$ days, peculiar SN Ia 2005hk at $t=+232$ days, and peculiar SN I 2008ha at $t=+65$ days. It is very unique that SN 2005cz shows only weak [O I] $\lambda \lambda 6300,6364$ and much stronger [Ca II] $\lambda \lambda 7291$, 7323 than $[\mathrm{O} \mathrm{I}]$.

\section{Faint Supernovae from $10-12 M_{\odot}$ Stars}

Kawabata et al. (2009) reported the unique properties of SN 2005cz, which provide a new clue to the understanding of the SN property-progenitor connection. SN $2005 \mathrm{cz}$ is a He-rich Type Ib SN (SN Ib) and appeared in the elliptical galaxy. This is peculiar because SN Ib is a core-collapse explosion of a He star and usually does not appear in elliptical galaxies that contain only old low-mass stars.

Further, SN 2005cz is unusually faint and rapidly fading (Fig. 2). The mass of ${ }^{56} \mathrm{Ni}$ is estimated to be $M\left({ }^{56} \mathrm{Ni}\right) \sim 0.018 \mathrm{M}_{\odot}$. The late-time spectrum of SN $2005 \mathrm{cz}$ at $t=+179$ days is very unique; unlike most of other SNe Ibc/IIb SN 2005cz shows much stronger [Ca II] than [O I] (Fig. 2) (Kawabata et al. 2009, Valenti et al. 2009, Foley et al. 2009).

Oxygen is ejected mostly from the oxygen layer formed during the hydrostatic burning phase. Thus its mass depends sensitively on the progenitor mass and is smaller for lowermass progenitors. On the other hand, Ca is explosively synthesized during the explosion. Theoretical models predict that the stars having main-sequence masses of $M_{\mathrm{ms}}=13 M_{\odot}$ and $18 M_{\odot}$ produce 0.2 and $0.8 M_{\odot}$ of $\mathrm{O}$, and 0.005 and $0.004 M_{\odot}$ of $\mathrm{Ca}$, respectively (Nomoto et al. 2006). Therefore, the $\mathrm{Ca} / \mathrm{O}$ ratio in the $\mathrm{SN}$ ejecta is sensitive to the progenitor mass. To produce the extremely large $\mathrm{Ca} / \mathrm{O}$ ratio, the mass of the progenitor star of SN $2005 \mathrm{cz}$ should be smaller than any other SNe Ib reported to date.

Kawabata et al. (2009) illustrate these unusual facts of SN $2005 \mathrm{cz}$ with the properties of SNe from the low-mass end of the core-collapse progenitors (i.e., either 8-10 $M_{\odot}$ or 10-12) in close binaries.

As for the host galaxy problem, the $10 M_{\odot}$ star model is found to be consistent with the properties recently-inferred for the host galaxy of SN 2005cz. It is still a genuine E2 galaxy, but has a relatively young stellar population with life times of $\sim 10^{7}-10^{8}$ years 

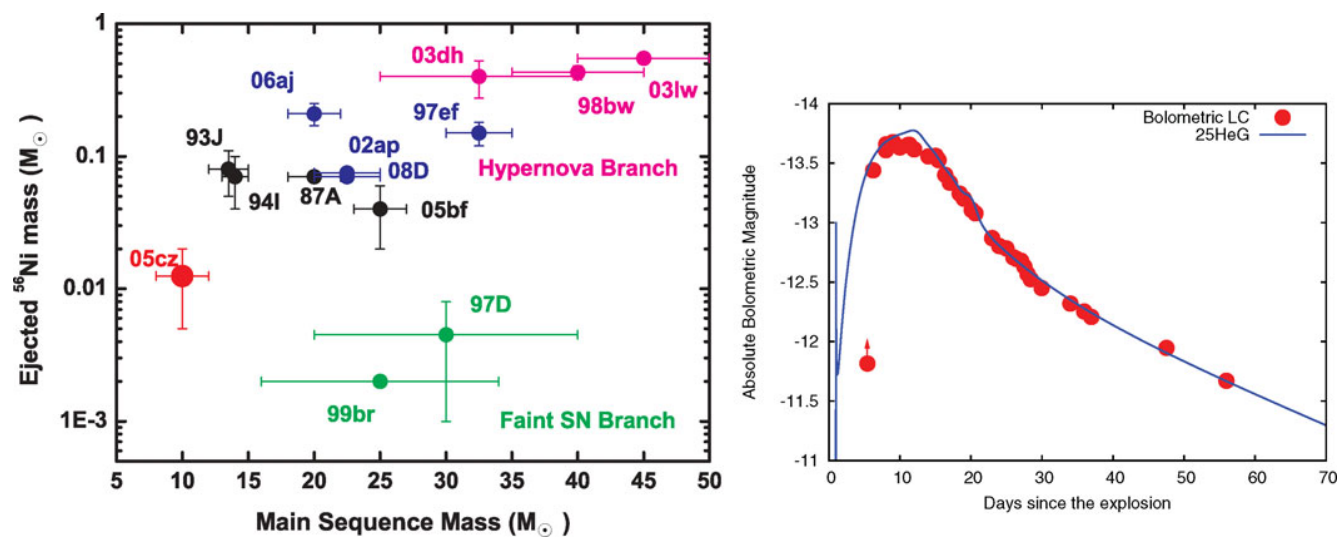

Figure 3. (Left): The ejected mass of ${ }^{56} \mathrm{Ni}$ as a function of the main sequence mass $M$ of the progenitors for several supernovae/hypernovae.

Figure 4. (Right:) The bolometric light curves of SN 2008ha and the model with $M=25 M_{\odot}$, $E=1.0 \times 10^{48} \mathrm{erg}, M_{\mathrm{ej}}=0.12 M_{\odot}$, and $0.003 M_{\odot}{ }^{56} \mathrm{Ni}$.

and SN Ib $2005 \mathrm{cz}$ is likely the end product of one of these young stars (see Kawabata et al. 2009 and references therein).

\section{Supernovae from $12 M_{\odot}-M_{\mathrm{BN}}$ Stars}

The supernova yields (including the mass of ${ }^{56} \mathrm{Ni}$ ) depend on the progenitor's mass $M$, metallicity, and the explosion energy $E$ (e.g., Kobayashi et al. 2006). From the comparison between the observed and calculated spectra and light curves of supernovae, we can estimate $M, E$, and the mass of ${ }^{56} \mathrm{Ni}$ as shown in Figure 3 (Nomoto et al. 2006, Kawabata et al. 2009). From this figure, the boundary mass between the NS and BH formation has been estimated to be $M_{\mathrm{BN}} \sim 25 M_{\odot}$. As shown in Nomoto et al. 2009, the yields between the three groups (Nomoto et al. 2006; Limongi et al. 2000; Heger \& Woosley 2008) are in good agreement for $M=15-25 M_{\odot}, E=1 \times 10^{51}$ erg and $Z=0.00-0.02$.

However, theoretical predictions of $\mathrm{Zn}, \mathrm{Co}, \mathrm{Ti} / \mathrm{Fe}$ are much smaller than those observed in extremely metal-poor (EMP) stars. The underproduction of these elements relative to $\mathrm{Fe}$ is much improved in the hypernova models (Fig. 5).

The abundance pattern of EMP stars in the Hercules dwarf spheroidal galaxy is very peculiar (Koch et al. 2008), but can be reproduced by yields of Hypernova model with $M=25 M_{\odot}$ and $E_{51}=20$ (Fig. 5; Tominaga et al. in prep.). These agreements suggest that hypernovae play an important role in the chemical enrichment during early galactic evolution.

\section{Hypernovae and Faint Supernovae from $M_{\mathrm{BN}}-90 M_{\odot}$ Stars}

$\mathrm{SNe}$ in this mass range form BHs and seem to bifurcate into the Hypernova branch and the Faint SNe branch (Fig. 3). The Hypernova branch include three SNe (1998bw, 2003dh, and 2003lw) that are associated with long Gamma-Ray Bursts (GRBs) (Fig. 3).

Among the Faint SNe, one of the faintest example is SN 2008ha (Valenti et al. 2009, Foley et al. 2009). This SN is of type I and the peak $V$ magnitude is only -14.2 mag. The rise and decline of the LC is quite fast. Line velocities are such low as 

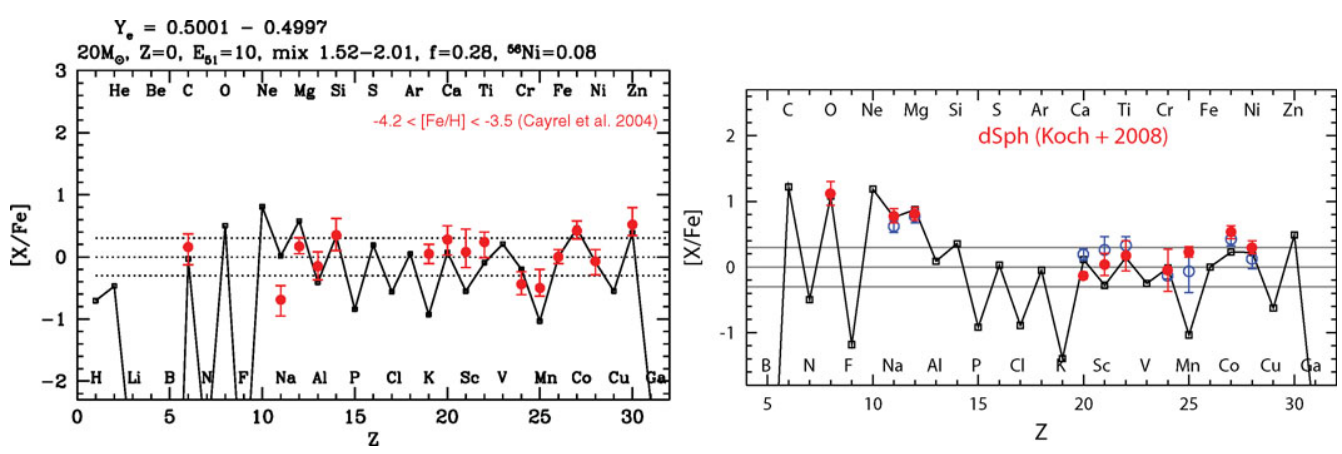

Figure 5. (Left): Averaged elemental abundances of stars with $[\mathrm{Fe} / \mathrm{H}]=-3.7$ (Cayrel et al. 2004) compared with the hypernova yield $\left(20 M_{\odot}, E_{51}=10\right)$.

Figure 6. (Right): The peculiar abundance pattern of the EMP stars in the Hercules dwarf spheroidal galaxy (Koch et al. 2008) is compared with the Hypernova yield (Tominaga et al. in prep.).

$\sim 2,000 \mathrm{~km} \mathrm{~s}^{-1}$. Moriya et al. (2009) have shown that these features can be reproduced by the core-collapse supernova model. Figure 3 shows the bolometric LC of the model with $M=25 M_{\odot}, E=1.0 \times 10^{48} \mathrm{erg}$, and $0.003 M_{\odot}{ }^{56} \mathrm{Ni}$. The ejecta of this explosion model undergoes large fallback because of low $E$, so that the ejecta mass is only $0.12 M_{\odot}$. The LC of this model well-reproduces SN 2008ha. (SN 2008ha is not included in Figure 3 because the model with $M=13 M_{\odot}$ and $E=1.4 \times 10^{48}$ erg also well-reproduces SN 2008ha (Moriya et al. 2009)).

The fallback SN (e.g., Iwamoto et al. 2005, Fryer et al. 2009) should also undergo mixing of ${ }^{56} \mathrm{Ni}$ before the occurrence of fallback in order to reproduce the observed light curve. Tominaga (2009) has shown that such "mixing and fallback" in spherical explosion is equivalent to the jet-induced nucleosynthesis.

In the jet-induced nucleosynthesis and mass ejection, the important parameter is the energy deposition rate $\dot{E}_{\text {dep }}$ (Tominaga et al. 2007). The variation of $\dot{E}_{\text {dep }}$ in the range of $\dot{E}_{\mathrm{dep}, 51} \equiv \dot{E}_{\mathrm{dep}} / 10^{51} \mathrm{ergs} \mathrm{s}^{-1}=0.3-1500$ leads to the following variation of the properties of GRBs and associated SNe. For low energy deposition rates $\left(\dot{E}_{\mathrm{dep}, 51}<3\right)$, the ejected ${ }^{56} \mathrm{Ni}$ masses $\left(M\left({ }^{56} \mathrm{Ni}\right)<10^{-3} M_{\odot}\right)$ are smaller than the upper limits for non-SN GRBs 060505 and 060614 (Iwamoto et al. 2005). For intermediate energy deposition rates $\left(3 \lesssim \dot{E}_{\mathrm{dep}, 51}<60\right)$, the explosions eject $10^{-3} M_{\odot} \lesssim M\left({ }^{56} \mathrm{Ni}\right)<0.1 M_{\odot}$, and the final $\mathrm{BH}$ masses are $10.8 M_{\odot} \lesssim M_{\mathrm{BH}}<15.1 M_{\odot}$. The resulting $\mathrm{SN}$ is faint $\left(M\left({ }^{56} \mathrm{Ni}\right)<0.01 M_{\odot}\right)$ or sub-luminous $\left(0.01 M_{\odot} \lesssim M\left({ }^{56} \mathrm{Ni}\right)<0.1 M_{\odot}\right)$.

Faint SN as a result of large fallback has been suggested to be responsible to produce the peculiar abundance patterns of extremely metal-poor (EMP) stars (Umeda \& Nomoto 2002, Iwamoto et al. 2005). In the jet-induced explosion model, the abundance patterns of EMP stars (esp. [C/Fe]) are related to $\dot{E}_{\text {dep }}$ as follows. Lower $\dot{E}_{\text {dep }}$ yields larger $M_{\mathrm{BH}}$ and thus larger $[\mathrm{C} / \mathrm{Fe}]$, because the infall reduces the amount of inner core material (Fe) relative to that of outer material $(\mathrm{C})$.

The observed abundance patterns of extremely metal-poor (EMP) stars are classified into three groups according to $[\mathrm{C} / \mathrm{Fe}]:(1)[\mathrm{C} / \mathrm{Fe}] \sim 0$, normal EMP stars $(-4<[\mathrm{Fe} / \mathrm{H}]$ $<-3$, e.g., Cayrel et al. 2004); (2) [C/Fe] +1 , Carbon-enhanced EMP (CEMP) stars $(-4<[\mathrm{Fe} / \mathrm{H}]<-3$, e.g., CS 22949-37, Depagne et al. 2002); (3) [C/Fe] +4, hyper 

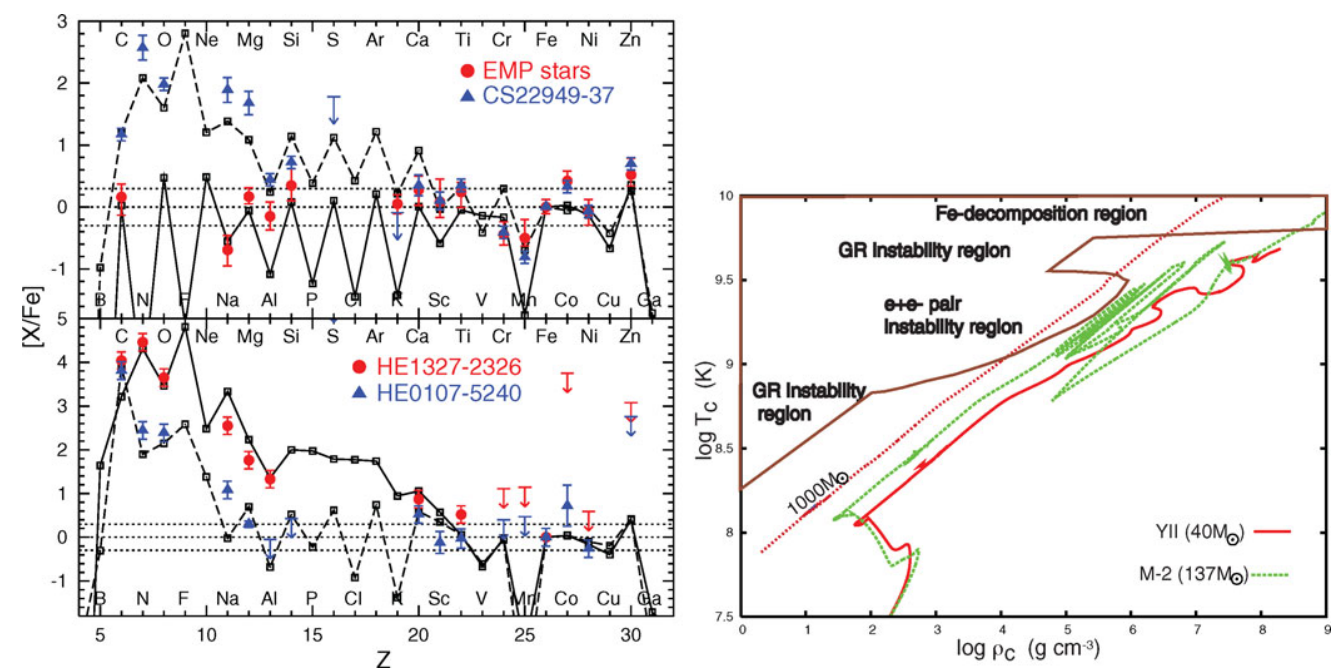

Figure 7. (Left): A comparison of the abundance patterns between metal-poor stars and models (Tominaga et al. 2007). Upper: typical EMP stars (red dots, Cayrel et al. 2004) and CEMP (blue triangles, CS 22949-37, Depagne et al. 2002) and models with $\dot{E}_{\mathrm{dep}, 51}=120$ (solid line) and $=3.0$ (dashed line). Lower: HMP stars: HE 1327-2326, (red dots, e.g., Frebel et al. 2005), and HE 0107-5240, (blue triangles, Christlieb et al. 2002, Bessell \& Christlieb 2005) and models with $\dot{E}_{\mathrm{dep}, 51}=1.5$ (solid line) and $=0.5$ (dashed line).

Figure 8. (Right): Evolutionary tracks of the central temperature and central density of very massive stars (Ohkubo et al. 2009). The numbers in brackets are the final masses for models YII and M-2. The $1000 M_{\odot}$ stars (Ohkubo et al. 2006) are also shown.

metal-poor (HMP) stars $([\mathrm{Fe} / \mathrm{H}]<-5$, e.g., HE 0107-5240, Christlieb et al. 2002; Bessell \& Christlieb 2005; HE 1327-2326, Frebel et al. 2005).

Figure 7 shows that the abundance patterns of the averaged normal EMP stars, the CEMP star CS 22949-37, and the two HMP stars (HE 0107-5240 and HE 1327-2326) are well reproduced by the models with $\dot{E}_{\mathrm{dep}, 51}=120,3.0,1.5$, and 0.5 , respectively. The model for the normal EMP stars ejects $M\left({ }^{56} \mathrm{Ni}\right) \sim 0.2 M_{\odot}$, i.e., a factor of 2 less than SN 1998bw. On the other hand, the models for the CEMP and the HMP stars eject $M\left({ }^{56} \mathrm{Ni}\right) \sim 8 \times 10^{-4} M_{\odot}$ and $4 \times 10^{-6} M_{\odot}$, respectively.

To summarize, (1) the explosions with large energy deposition rate, $\dot{E}_{\mathrm{dep}}$, are observed as GRB-HNe, and their yields can explain the abundances of normal EMP stars, and (2) the explosions with small $\dot{E}_{\text {dep }}$ are observed as GRBs without bright SNe and can be responsible for the formation of the CEMP and the HMP stars. We thus propose that GRB-HNe and GRBs without bright SNe belong to a continuous series of BH-forming massive stellar deaths with relativistic jets of different $\dot{E}_{\mathrm{dep}}$.

\section{Luminous Supernovae from $90-140 M_{\odot}$ Stars}

Massive Pop III stars are formed through mass accretion, starting from a tiny core through collapse (e.g., Yoshida et al. 2008). Such an evolution with mass accretion starting from $M \sim 1 M_{\odot}$ has recently been studied by Ohkubo et al. $(2006,2009)$. Figure 5 shows the evolutionary tracks of the central density and temperature in the later phases.

The star M-2, whose final mass is $137 M_{\odot}$, undergoes nuclear instability due to oxygen and silicon burning and pulsates (Nomoto et al. 2005; Woosley et al. 2007; Umeda \& 

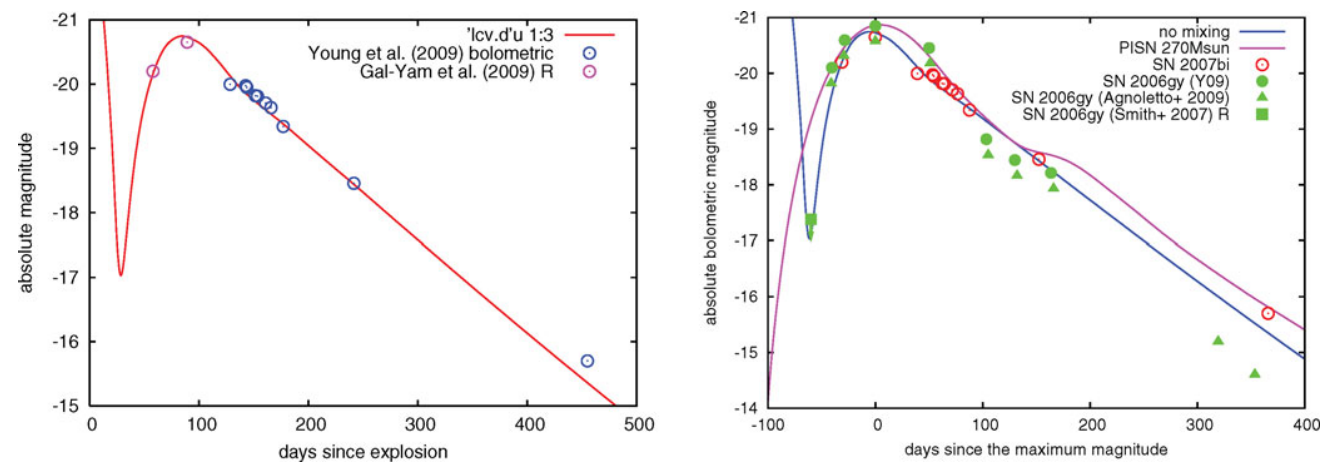

Figure 9. (Left): The bolometric light curve of the $\mathrm{C}+\mathrm{O}$ core $\mathrm{SN}$ models $\left(M_{\mathrm{ej}}=39 M_{\odot}, E_{\mathrm{kin}}=3.3 \times 10^{52} \mathrm{erg}\right.$, and $\left.M\left({ }^{56} \mathrm{Ni}\right)=6.1 M_{\odot}\right)$ compared with the bolometric LC of SN 2007bi (Moriya et al. 2009).

Figure 10. (Right): The PISN model $\left(M=270 M_{\odot}\right)$ for the LC of SNe 2006gy and 2007bi (Moriya et al. 2009).

Nomoto 2008; Ohkubo et al. 2009). In the extreme case, the pulsation could induce dynamical mass ejection and optical brightening as might be observed in the brightest SN 2006gy (Woosley et al. 2007).

If the explosion energy is large enough, the mass of ${ }^{56} \mathrm{Ni}$ can be as large as $\sim 6 M_{\odot}$ (Umeda \& Nomoto 2008). The resultant light curve can be consistent with Luminous Supernovae such as SNe 2006gy and 2007bi (Figs. 7 and 7: Moriya et al. 2009).

\section{Pair-Instability Supernovae from 140 - $300 M_{\odot}$ Stars}

These very massive stars (VMS) undergo pair-creation instability and are disrupted completely by explosive oxygen burning, as pair-instability supernovae (PISNe) (e.g., Barkat et al. 1967; Arnett 1996; Umeda \& Nomoto 2002; Heger \& Woosley 2002).

Their LCs can be consistent with LSNe 2007bi and 2006gy (Gal-Yam et al. 2009) as seen in Figs. 7 and 7 (Moriya et al. 2009).

However the abundance patterns of the ejected material for the $200 M_{\odot}$ star (Umeda \& Nomoto 2002) are compared with EMP stars. It is clear that PISN ejecta cannot be consistent with the large $\mathrm{C} / \mathrm{Fe}$ observed in HMP stars and other C-rich EMP stars. Also, the abundance ratios of iron-peak elements $([\mathrm{Zn} / \mathrm{Fe}]<-0.8$ and $[\mathrm{Co} / \mathrm{Fe}]<-0.2)$ in the PISN ejecta cannot explain the large $\mathrm{Zn} / \mathrm{Fe}$ and $\mathrm{Co} / \mathrm{Fe}$ ratios in typical EMP stars.

\section{Very Massive Stars $\left(>300 M_{\odot}\right)$ and Intermediate Mass Black Holes}

It is possible that the First Stars were even more massive than $\sim 300 M_{\odot}$, if rapid mass accretion continues during the whole main-sequence phase of Pop III stars (Ohkubo et al. 2006, 2008).

Such massive stars undergo core-collapse (CVMS: core-collapse VMS) as seen from the $1000 M_{\odot}$ star track in Figure 5. If such stars formed rapidly rotating black holes, jet-like mass ejection could produce processed material (Ohkubo et al. 2006). In fact, for moderately aspherical explosions, the patterns of nucleosynthesis match the observational data of both intracluster medium and M82 (Ohkubo et al. 2006). 
It is also possible that LSNe 2006gy and/or 2007bi can be the explosion of the above CVMS.

This result suggests that explosions of CVMS contribute significantly to the chemical evolution of gases in clusters of galaxies. This result may support the view that Pop III CVMS could be responsible for the origin of intermediate mass black holes (IMBH).

\section{References}

Arnett, W. D. 1996, Supernovae and Nucleosynthesis (Princeton: Princeton Univ. Press)

Barkat, Z., Rakavy, G., \& Sack, N. 1967, PRL 18, 379

Bessell, M. S. \& Christlieb, N. 2005, in V. Hill et al. (eds.), From Lithium to Uranium, Proc. IAU Symposium No. 228 (Cambridge: Cambridge Univ. Press), 237

Cayrel, R., et al. 2004, A\&SA 416, 1117

Christlieb, N., et al. 2002, Nature 419, 904

Depagne, E., et al. 2002, A\&A 3 390, 187

Foley, R. J., et al. 2009, AJ 138, 376

Frebel, A., et al. 2005, Nature 434, 871

Fryer, C., et al. 2009, arXiv:0908.0701

Gal-Yam, A., et al. 2009, Nature 462, 624

Heger, A. \& Woosley, S. E. 2002, ApJ 567, 532

Heger, A. \& Woosley, S. E. 2008, ApJ submitted (arXiv:0803.3161)

Iwamoto, K., Mazzali, P. A., Nomoto, K., et al. 1998, Nature 395, 672

Iwamoto, N., Umeda, H., Tominaga, N., Nomoto, K., \& Maeda, K. 2005, Science 309, 451

Kawabata, K., Maeda, K., Nomoto, K., et al. 2009, arXiv:0906.4573

Kitaura, F. S., Janka, H.-Th., \& Hillebrandt, W. 2006, A $\mathscr{E} A$ 450, 345

Koch, A., et al. 2008, ApJ 688, L13

Kobayashi, C., Umeda, H., Nomoto, K., Tominaga, N., \& Ohkubo, T. 2006, ApJ 653, 1145

Limongi, M., Straniero, O., \& Chieffi, A. 2000, ApJS 129, 625

Maeda, K. \& Nomoto, K. 2003, ApJ 598, 1163

Moriya, T., et al. 2009, ApJ submitted

Nomoto, K. 1984, ApJ 277, 791

Nomoto, K. \& Hashimoto, M. 1988, Phys. Rep. 163, 13

Nomoto, K., et al. 2005, in The Fate of Most Massive Stars, ed. R. Humphreys \& K. Stanek (ASP Ser. 332), 374 (astro-ph/0506597)

Nomoto, K., et al. 2006, Nuclear Phys A 777, 424 (astro-ph/0605725)

Nomoto, K., et al. 2009, in IAU Symp. 254, The Galaxy Disk in Cosmological Context, ed. J. Andersen, et al. (Cambridge: Cambridge Univ. Press), 355 (arXiv: 0901.4536)

Ohkubo, T., Umeda, H., Maeda, K., Nomoto, K., Suzuki, T., Tsuruta, S., \& Rees, M. J. 2006, ApJ 645, 1352

Ohkubo, T., Nomoto, K., Umeda, H., Yoshida, N., \& Tsuruta, S. 2009, ApJ 706, 1184

Prieto, J. L., et al. 2008, ApJ 681, L9

Pumo, M. L., et al. 2009, ApJ 705, L138

Smartt, S. J. 2009, ARA $\& A 47,63$

Thompson, T. A., et al. 2009, ApJ 705, 1364

Tominaga, N., Maeda, K., Umeda, H., Nomoto, K., Tanaka, et al. 2007, ApJ 657, L77

Tominaga, N. 2009, ApJ 690, 526

Umeda, H. \& Nomoto, K. 2002, ApJ 565, 385

Umeda, H. \& Nomoto, K. 2008, ApJ 673, 1014

Valenti, S., et al. 2009, Nature 459, 674

Wanajo, S., Nomoto, K., Janka, H.-T., Kitaura, F. S., \& Müller, B. 2009, ApJ 695, 208

Woosley, S. E., Blinnikov, S., \& Heger, A. 2007, Nature 450, 390

Young, D. R., et al. 2009, arXiv:0910.2248

Yoshida, N., Omukai, K., \& Hernquist, L. 2008, Science 321, 669 